Pacific Journal of Mathematic 


\title{
DIMENSION THEORY IN POWER SERIES RINGS
}

\author{
DAVID E. FIELDS
}

Let $V$ be a valuation ring of finite rank $n$. If $V$ is discrete, then $V[[X]]$ has dimension $n+1$. If $V$ is not discrete, then the dimension of $V[[X]]$ is at least $n+k+1$, where $k$ is the number of idempotent proper prime ideals of $V$.

Let $R$ be a commutative ring with identity. If there exists a chain $P_{0} \subset P_{1} \subset P_{2} \subset \cdots \subset P_{n}$ of $n+1$ prime ideals of $R$, where $P_{n} \subset R$, but no such chain of $n+2$ prime ideals, then we say that $R$ has dimension $n$ and we write $\operatorname{dim} R=n$ [3]. In [3] and [4], Seidenberg has investigated the dimension theory of $R\left[X_{1}, X_{2}, \cdots, X_{m}\right]$ where $R$ has finite dimension and $X_{1}, X_{2}, \cdots, X_{m}$ are indeterminates over $R$. We investigate the dimension theory of $V[[X]]$ where $V$ is a valuation ring.

Throughout this paper, $R$ denotes a commutative ring with identity; $\omega$ is the set of natural numbers; $\omega_{0}$ is the set of nonnegative integers; and $Z$ is the set of integers. If

$$
f(X)=\sum_{i=0}^{\infty} f_{i} X^{i} \in R[[X]],
$$

we denote by $A_{f}$ the ideal of $R$ generated by the coefficients of $f(X): A_{f}=\left\{f_{0}, f_{1}, \cdots, f_{k}, \cdots\right\} R$. If $A$ is an ideal of $R$, we let

$$
A[[X]]=\left\{f(X)=\sum_{i=0}^{\infty} f_{i} X^{i}: f_{i} \in A \text { for each } i \in \omega_{0}\right\}
$$

and we define $A \cdot R[[X]]$ to be the ideal of $R[[X]]$ which is generated by $A$. Then $A \cdot R[[X]]=\left\{f(X): A_{f} \subseteq B\right.$ for some finitely generated ideal $B$ of $R$ with $B \cong A\}$. It is clear that $A \cdot R[[X]] \subseteq$ $A[[X]]$; equality holds if and only if each countably generated ideal of $R$ contained in $A$ is contained in a finitely generated ideal of $R$ contained in $A$. In particular, if $V$ is a valuation ring containing an ideal $A$ which is countably generated but not finitely generated, then $A \cdot V[[X]] \subset A[[X]]$. Finally, we note that if $A$ is an ideal of $R$, then $R[[X]] / A[[X]] \cong(R / A)[[X]]$; hence $A[[X]]$ is a prime ideal of $R[[X]]$ if and only if $A$ is a prime ideal of $R$.

2. Discrete valuation rings, Let $V$ be a valuation ring of rank $k$ with associated valuation $v$ and value group $G$; let $\{0\}=$ $G_{0} \subset G_{1} \subset \cdots \subset G_{k}=G$ be the chain of isolated subgroups of $G$ together with $G$. In [2], Iwasawa proves that for $1 \leqq i \leqq k$, 
$G_{i} / G_{i-1} \cong H_{i}$ where $H_{i}$ is a subgroup of the additive group of real numbers, this being an order-preserving isomorphism of groups. If for $1 \leqq i \leqq k, H_{i} \cong Z$, we shall say that $V$ is a discrete valuation ring of rank $k$. This is equivalent to the condition that $V$ contains no idempotent proper prime ideal.

LEMma 2.1. Let $V$ be a valuation ring and let $P$ be a proper prime ideal of $V$. If $P$ is not idempotent, then in $V[[X]]$, $\sqrt{(P \cdot V[[X]])}=P[[X]]$ and $(P[[X]])^{2} \subseteq P \cdot V[[X]]$.

Proof. Let $\alpha \in P, \alpha \notin P^{2}$. Then

$$
(P[[X]])^{2} \subseteq P^{2}[[X]] \subseteq(\alpha) V[[X]] \subseteq P \cdot V[[X]] \cdot
$$

Hence $P[[X]] \subseteq \sqrt{(P \cdot V[[X]])}$ and the reverse containment is clear.

Lemma 2.2. Let $V$ be a valuation ring with quotient field $K$ and let $P$ be a proper prime ideal of $V$. Let

$$
D=V[[X]][K]=(V[[X]])_{V \backslash\{0\}} .
$$

Then $D=\left(V_{P}[[X])_{V_{P} \backslash\{0\}}\right.$.

Proof. We first show that $V_{P}[[X]] \subseteq D$. Let

$$
f(X)=\sum_{i=0}^{\infty} f_{i} X^{i} \in V_{P}[[X]]
$$

For each $i \in \omega_{0}$, there exists $r_{i} \in V \backslash P$ such that $r_{i} f_{i} \in V$. Let $a \in P \backslash\{0\}$; then for each $i \in \omega_{0}, a / r_{i} \in P V_{P}=P \subseteq V$, implying that $a f_{i}=\left(a / r_{i}\right)\left(r_{i} f_{i}\right) \in V$; that is, $a f(X) \in V[[X]]$. This implies that $f(X) \in(V[[X]])_{V \backslash(0)}=D$, showing that $V_{P}[[X]] \subseteq D$.

Since $D \supseteqq K$, each nonzero element of $V_{P}$ is a unit in $D$. Thus $D \supseteqq\left(V_{P}[[X]]\right)_{V_{P} \backslash\{0\}}$ and the reverse containment is obvious.

COROLlaRY 2.3. Let $V$ be a valuation ring and let $P$ be a proper prime ideal of $V$. There is a one-to-one correspondence between prime ideals of $V[[X]]$ which contract to $(0)$ in $V$ and prime ideals of $V_{P}[[X]]$ which contract to $(0)$ in $V_{P}$; this correspondence preserves containment.

Proof. Lemma 2.2 assures that there is a one-to-one, containment preserving correspondence between each of these classes of prime ideals and the class of prime ideals of $D$.

LEMmA 2.4. Let $R$ be a quasi-local ring having maximal ideal 
$M$. Let $f(X) \in R[[X]], \quad f(X) \notin M[[X]]-$ say $f_{k} \in R \backslash M, k$ minimal. There exists $g(X)$, a unit of $R[[X]]$, such that $f(X) g(X)$ has exactly one unit coefficient, namely $(f g)_{k}$.

Proof. For $u(X) \in R[[X]]$, denote by $\bar{u}(X)$ the canonical image of $u(X)$ in $(R / M)[[X]]$. By choice of $k$,

$$
\bar{f}(X)=\bar{f}_{k} X^{k}+\bar{f}_{k+1} X^{k+1}+\cdots=X^{k}\left(\bar{f}_{k}+\bar{f}_{k+1} X+\cdots\right),
$$

where $\bar{f}_{k} \neq 0$. Then $\bar{f}_{k}+\bar{f}_{k+1} X+\cdots$ is a unit of $(R / M)[[X]]$, and we can choose $g(X) \in R[[X]]$ such that $\bar{g}(X) \cdot\left(\bar{f}_{k}+\bar{f}_{k+1} X+\cdots\right)=1$. Thus $\bar{f}(X) \cdot \bar{g}(X)=X^{k}$, and $f(X) g(X)-X^{k} \in M[[X]]$. This implies that only the coefficient of $X^{k}$ in $f(X) g(X)$ is not in $M$.

Corollary 2.5. Let $V$ be a valuation ring and let $P$ be a proper prime ideal of $V$. If $Q$ is an ideal of $V_{P}[[X]]$ and if $Q \nsubseteq\left(P V_{P}\right)[[X]]$, then $Q \cap V[[X]] \nsubseteq P[[X]]$.

Proof. Lemma 2.4 assures that there is a power series $g(X)$ in $Q$ with $g(X)$ having exactly one unit coefficient, $g_{k}$. Since $g_{k}$ is a unit of $V_{P}$, there in no loss of generality in assuming that, in fact, $g_{k}=1$. Then for $i \neq k, \quad g_{i} \in P V_{P}=P \leqq V$, implying that $g(X) \in Q \cap V[[X]]$ while $g(X) \notin P[[X]]$.

Lemma 2.6. ${ }^{1}$ Let $R$ be a Noetherian ring having dimension $n$. Then $R\left[\left[X_{1}, X_{2}, \cdots, X_{m}\right]\right]$ is Noetherian and has dimension $n+m$.

Proof. It is well known that if $R$ is Noetherian, then $R\left[\left[X_{1}, X_{2}, \cdots, X_{m}\right]\right]$ is Noetherian. We shall show that the dimension of $R[[X]]$ is $n+1$; the lemma follows immediately by induction on $m$.

Let $M$ be a maximal ideal of $R[[X]]$. Then $M=M_{1}+(X)$ for some maximal ideal $M_{1}$ of $R$. Since $\operatorname{dim} R=n$, the height of $M_{1}$ is $k$ where $k \leqq n$. There exists an ideal $A=\left(a_{1}, a_{2}, \cdots, a_{k}\right)$ of $R$ which admits $M_{1}$ as an isolated prime ideal [5;242]. It is straightforward to verify that $M=M_{1}+(X)$ is an isolated prime ideal of $A+(X)=$ $\left(a_{1}, a_{2}, \cdots, a_{k}, X\right) R[[X]]$. This implies that the height of $M$ is at most $k+1$ [5;240]; since $k \leqq n$, the height of $M$ is at most $n+1$. Since $M$ was an arbitrary maximal ideal of $R[[X]]$, we conclude that $\operatorname{dim} R[[X]] \leqq n+1$; the reverse inequality is clear.

THEOREM 2.7. Let $V$ be a discrete valuation ring of rank $n$ and let $(0)=P_{0} \subset P_{1} \subset P_{2} \subset \cdots \subset P_{n}$ be the nonunit prime ideals of

\footnotetext{
1 The proof of Lemma 2.6 was pointed out to the author by William Heinzer.
} 
V. Then $\operatorname{dim} V[[X]]=n+1$.

Proof. We use induction on $n$, the case $n=1$ following from Lemma 2.6 since a rank one discrete valuation ring is Noetherian.

Assuming the result for discrete valuation rings of rank less than $n$, let $V$ be a discrete valuation ring of rank $n$ and let $(0) \subset Q_{1} \subset Q_{2} \subset \cdots \subset Q_{t}$ be a chain of prime ideals of $V$ [[X]]. We consider two cases.

Case 1. $Q_{1} \cap V \neq(0)$. Here $Q_{1} \cap V \supseteqq P_{1}$, so that $Q_{1} \supseteqq P_{1} \cdot V[[X]]$, implying that $Q_{1} \supseteqq \sqrt{\left(P_{1} \cdot V[[X]]\right)}=P_{1}[[X]]$, the latter equality being a consequence of Lemma 2.1. But the depth of $P_{1}[[X]]$ cannot exceed $\operatorname{dim}\left(V / P_{1}\right)[[X]]=n$; we conclude that $t \leqq n+1$.

Case 2. $Q_{1} \cap V=(0)$. Corollary 2.3 asserts that $Q_{1}=Q^{*} \cap V[[X]]$, where $Q^{*}$ is a prime ideal of $V_{P_{1}}[[X]]$ and $Q^{*} \cap V_{P_{1}}=(0)$. Since $\operatorname{dim} V_{P_{1}}[[X]]=2, Q^{*} \nsubseteq\left(P_{1} V_{P_{1}}\right)[[X]]$. By Corollary 2.5, $Q_{1} \nsubseteq P_{1}[[X]]$. Since $V_{P_{1}}[[X]]$ is two-dimensional and local, each proper prime ideal of $V_{P_{1}}[[X]]$ which contracts to (0) in $V_{P_{1}}$ is a minimal prime ideal of $V_{P_{1}}[[X]]$. Corollary 2.3 now assures that each proper prime ideal of $V$ [[X]] which contracts to $(0)$ in $V$ is a minimal prime ideal of $V[[X]]$. It follows that $Q_{2} \cap V \neq(0)$, implying that $Q_{2} \supseteqq P_{1}[[X]]$. Since also $Q_{2} \supseteqq Q_{1}$ and $Q_{1} \nsubseteq P_{1}[[X]]$, we conclude that $Q_{2} \supset P_{1}[[X]]$. Thus we have a chain $(0) \subset P_{1}[[X]] \subset Q_{2} \subset Q_{3} \subset \cdots \subset Q_{t}$. It follows, as in Case 1 , that $t \leqq n+1$.

Thus $\operatorname{dim} V[[X]] \leqq n+1$ and the reverse inequality is clear.

3. Rank one nondiscrete valuation rings. We note that if $V$ is a rank one valuation ring, then the value group of $v$ is Archimedian.

Lemma 3.1. Let $V$ be a valuation ring and let $B$ be an ideal of $V$. If $B$ is not finitely generated, then the following conditions are equivalent:

(a) $f(X) \in B \cdot V[[X]]$.

(b) $A_{f} \subseteq(b)$ for some $b \in B$.

(c) $f(X)=b g(X)$ for some $b \in B, g(X) \in V[[X]]$.

(d) $A_{f} \subset B$.

Proof. We establish that $(\mathrm{a}) \rightarrow(\mathrm{b}) \rightarrow(\mathrm{c}) \rightarrow(\mathrm{a})$ and that $(\mathrm{b}) \leftrightarrow(\mathrm{d})$. $(\mathrm{a}) \rightarrow(\mathrm{b})$ : Let $f(X) \in B \cdot V[[X]]$; then we can write

$$
f(X)=b_{1}\left[g^{(1)}(X)\right]+b_{2}\left[g^{(2)}(X)\right]+\cdots+b_{t}\left[g^{(t)}(X)\right]
$$


where for $1 \leqq i \leqq t, b_{i} \in B$ and $g^{(i)}(X)=\sum_{j=0}^{\infty} g_{i j} X^{j} \in V[[X]]$. Thus $f(X)=\sum_{i=0}^{\infty} f_{i} X^{i}$ where $f_{i}=\sum_{k=1}^{t} b_{k} g_{k i}$. In $V,\left(b_{1}, b_{2}, \cdots, b_{t}\right)=\left(b_{s}\right)$ for some $s, 1 \leqq s \leqq t$. Now for $i \in \omega_{0}, f_{i}=\sum_{k=1}^{t} b_{k} g_{k i} \in\left(b_{s}\right)$, implying that $A_{f} \leqq\left(b_{s}\right)$ where $b_{s} \in B$.

$(b) \rightarrow(c)$ : We assume that $A_{f} \subseteq(b)$; then for $i \in \omega_{0}, f_{i}=b g_{i}$ where $g_{i} \in V$. Let $g(X)=\sum_{i=0}^{\infty} g_{i} X^{i}$; it then is clear that $f(X)=$ $b g(X)$.

(c) $\rightarrow($ a): This is obvious.

(b) $\rightarrow(\mathrm{d})$ : This is immediate from the assumption that $B$ is not finitely generated.

$(\mathrm{d}) \rightarrow(\mathrm{b})$ : Assuming that $A_{f} \subset B$, let $b \in B, \quad b \notin A_{f}$. Then (b) $\nsubseteq A_{f}$ so $A_{f} \leqq(b)$ since $V$ is a valuation ring.

THEOREM 3.2. Let $V$ be a rank one nondiscrete valuation ring having maximal ideal $M$. Then $M \cdot V[[X]]=\sqrt{(M \cdot V[[X]])}$.

Proof. Let $f(X) \in \sqrt{(M \cdot V[[X]])}$ - say $[f(X)]^{k} \in M \cdot V[[X]]$; we then can write $[f(X)]^{k}=r g(X)$ where $r \in M$ and $g(X) \in V[[X]]$. There exists an element $s$ of $M$ with $0<v(s) \leqq v(r) / k$; then $r=s^{k} t$ where $t \in V$, implying that $[f(X)]^{k}=r g(X)=s^{k} t g(X)$, so that

$$
[f(X)]^{k} / s^{k}=[f(X) / s]^{k}=\operatorname{tg}(X) \in V[[X]] \text {. }
$$

Therefore $f(X) / s$ is a root of $Z^{k}-t g(X) \in V[[X]][Z]$, whereby $f(X) / s$ is integral over $V[[X]]$. Also $f(X) / s$ clearly is in the quotient field of $V[[X]]$. But $V$ is completely integrally closed, implying that $V[[X]]$ is completely integrally closed, hence is integrally closed $[1 ; 150]$. Thus $f(X) / s=h(X) \in V[[X]]$ and $f(X)=$ $\operatorname{sh}(X) \in M \cdot V[[X]]$ since $s \in M$. Hence $\sqrt{(M \cdot V[[X]])} M \cdot V[[X]]$, so that equality holds.

THEOREM 3.3. Let $R$ be a quasi-local ring having maximal ideal $M$ and let $Q$ be a prime ideal of $R[[X]]$. If $Q \supseteqq M \cdot R[[X]]$, then either $Q \supseteqq M[[X]]$ or $Q \subseteq M[[X]]$.

Proof. We assume that $Q \nsubseteq M[[X]]$ and show that $Q \supseteqq M[[X]]$. Let $f(X)=\sum_{i=0}^{\infty} f_{i} X^{i} \in Q, f(X) \notin M[[X]]$. Let $t$ be the smallest integer $k$ for which $f_{k}$ is a unit of $R$. Let $g(X)=\sum_{i=0}^{t-1} f_{i} X^{i}$ if $t>0$; let $g(X)=0$ if $t=0$. Then $g(X) \in M \cdot R[[X]] \subseteq Q$, implying that $f(X)-g(X) \in Q$. If $f(X)-g(X)$ has order zero, then $g(X)=0$, so that $f_{0}$ is a unit of $R$, implying that $f(X)$ is a unit of $R[[X]]$, whence $Q=R[[X]] \supseteqq M[[X]]$. If $f(X)-g(X)$ has positive order $n$, then $[f(X)-g(X)]_{n}$ is a unit of $R$ and $f(X)-g(X)=X^{n} h(X)$ where $h_{0}=[f(X)-g(X)]_{n}$ is a unit of $R$, implying that $h(X)$ is a unit of $R[[X]]$. 
Since $f(X)-g(X)=X^{n} h(X) \in Q$ and $Q$ is a prime ideal of $R[[X]]$, either $X^{n} \in Q$ or $h(X) \in Q$. If $X^{n} \in Q$, then $X \in Q$, implying that $Q \supseteqq M \cdot R[[X]]+(X) \supseteqq M[[X]]$. If $h(X) \in Q$, then $Q=R[[X]] \supseteqq$ $M[[X]]$. Hence if $Q \nsubseteq M[[X]]$, then $Q \supseteqq M[[X]]$.

THEOREM 3.4. Let $V$ be a rank one nondiscrete valuation ring having maximal ideal $M$.

(a) There is a prime ideal $P$ of $V[[X]]$ satisfying $M \cdot V[[X]] \subseteq$ $P \subset M[[X]]$.

(b) $\operatorname{dim} V[[X]] \geqq 3$.

Proof. Theorem 3.2 asserts that

$$
\sqrt{(M \cdot V[[X]])}=M \cdot V[[X]] \subset M[[X]] \cdot
$$

Hence there is a prime ideal $P$ of $V[[X]]$ satisfying $P \supseteqq M \cdot V[[X]]$, $P \nsupseteq M[[X]]$. Theorem 3.3 then asserts that $P \subset M[[X]]$; hence (a) holds.

We now have a chain $(0) \subset P \subset M[[X]] \subset M \cdot V[[X]]+(X)$ of prime ideals of $V[[X]]$, implying $(b)$.

4. Valuation rings of finite rank.

LemmA 4.1. Let $V$ be a valuation ring and let $P$ be a proper prime ideal of $V$. Then $P V_{P}=P$; hence $P$ is idempotent if and only if $P V_{P}$ is idempotent.

The proof of Lemma 4.1 is straightforward and will therefore be omitted.

LEMMA 4.2. Let $V$ be a valution ring and let $P$ be an idempotent proper prime ideal of $V$. Then $P \cdot V[[X]]=\left(P V_{P}\right) \cdot V_{P}[[X]]$.

Proof. Let $f(X) \in\left(P V_{P}\right) \cdot V_{P}[[X]]$ - say $f(X)=r h(X)$ where $r \in P V_{P}$ and $h(X) \in V_{P}[[X]]$. Since $P=P V_{P}$ is idempotent, we can write $r=s t$ where $s, t \in P=P V_{P}$; then for $i \in \omega_{0}$, there exists $a_{i} \in V \backslash P$ such that $a_{i} h_{i} \in V$. Since $a_{i} \in V \backslash P$ and $t \in P$, we have that $(t) \leqq\left(a_{i}\right)$ so that $t / a_{i} \in V$ for each $i \in \omega_{0}$, implying that $t h_{i}=\left(t / a_{i}\right)\left(a_{i} h_{i}\right) \in V$ for each $i \in \omega_{0}$ - that is, $t h(X) \in V[[X]]$. Since $s \in P$, we conclude that $f(X)=r h(X)=s(\operatorname{th}(X)) \in P \cdot V[[X]]$, establishing that

$$
\left(P V_{P}\right) \cdot V_{P}[[X]] \subseteq P \cdot V[[X]] \cdot
$$

The reverse containment is obvious. 
THEOREM 4.3. Let $V$ be a valuation ring and let $P$ be a proper prime ideal of $V$. If $Q$ is a prime ideal of $V[[X]]$ and if $Q \supseteqq P$. $V[[X]]$, then either $Q \supseteqq P[[X]]$ or $Q \subseteq P[[X]]$.

Proof. Assuming that $Q \nsubseteq P[[X]]$, we first establish that either $X \in Q$ or $Q$ contains $h(X)$, where $h(X) \in V[[X]]$ and $h_{0} \notin P$. Let $f(X)=\sum_{i=0}^{\infty} f_{i} X^{i} \in Q, f(X) \notin P[[X]]$. Let $t$ be the smallest integer $k$ for which $f_{k} \notin P$. If $t=0$, then we let $h(X)=f(X)$. If $t>0$, then we let $g(X)=\sum_{i=0}^{t-1} f_{i} X^{i}$. Then $g(X) \in P \cdot V[[X]] \subseteq Q$, implying that $f(X)-g(X) \in Q$. Further, $f(X)-g(X)=X^{t} h(X)$ where $h_{0}=f_{t} \notin P$. Since $Q$ is prime, either $X \in Q$ or $h(X) \in Q$. Hence if $Q \nsubseteq P[[X]]$, then either $X \in Q$ or $Q$ contains $h(X)$ where $h(X) \in$ $V[[X]]$ and $h_{0} \notin P$.

If $X \in Q$, then $Q \supseteqq P[[X]]$; hence we consider the case where $h(X) \in Q$ with $h_{0} \notin P$. Observe now that $h(X) \in V_{P}[[X]]$ and that $h_{0}$ is a unit of $V_{P}$, implying that $h(X)$ is a unit of $V_{P}[[X]]-$ that is $1 / h(X) \in V_{P}[[X]]$. Now let $r(X) \in P[[X]]$; then

$$
r(X)[1 / h(X)] \in P[[X]] \cdot V_{P}[[X]] \subseteq P[[X]]
$$

- in particular, $r(X)[1 / h(X)] \in V[[X]]$. Since $h(X) \in Q$, we see that $r(X)=h(X)[r(X) / h(X)] \in Q$. Hence $Q \supseteqq P[[X]]$.

Lemma 4.4. Let $V$ be a valuation ring having a minimal prime ideal $P$. If $P$ is idempotent, then $P \cdot V[[X]]=\sqrt{(P \cdot V[[X]])}$.

Proof. Let $f(X) \in \sqrt{(P \cdot V[[X]])}$. Then in

$$
V_{P}[[X]], f(X) \in \sqrt{\left(\left(P V_{P}\right) \cdot V_{P}[[X]]\right)}
$$

by Lemma 4.2. Since $V_{P}$ is a rank one nondiscrete valuation ring, Theorem 3.2 asserts that $\sqrt{\left(\left(P V_{P}\right) \cdot V_{P}[[X]]\right)}=\left(P V_{P}\right) \cdot V_{P}[[X]]$. Hence $f(X) \in\left(P V_{P}\right) \cdot V_{P}[[X]]=P \cdot V[[X]]$, the latter equality following from Lemma 4.2.

THEOREM 4.5. Let $V$ be a valuation ring and let $P$ be a proper prime ideal of $V$. If $P$ is idempotent, then

$$
P \cdot V[[X]]=\sqrt{(P \cdot V[[X]])} \text {. }
$$

Proof. We shall say that $P$ is branched provided there exists a $P$-primary ideal distinct from $P[1 ; 173]$. We consider two cases.

Case 1. $P$ is branched. Then there is a prime ideal $Q$ of $V$ with $Q \subset P$ and such that there are no prime ideals of $V$ properly 
between $Q$ and $P[1 ; 173]$. Then $P / Q$ is a minimal prime ideal of $V / Q$ and $P / Q$ is idempotent. Lemma 4.4 assures that

$$
(P / Q) \cdot(V / Q)[[X]]=\sqrt{((P / Q) \cdot(V / Q)[[X]])} .
$$

By considering the natural homomorphism from $V[[X]]$ to $(V / Q)[[X]]$, we conclude that $P \cdot V[[X]]=\sqrt{(P \cdot V[[X]])}$.

Case 2. $P$ is not branched. Then $P=\mathrm{U}_{\lambda} M_{\lambda}$ where $\left\{M_{\lambda}\right\}_{\lambda \in A}$ is the collection of prime ideals of $V$ properly contained in $P[1 ; 173]$. Let $f(X) \in \sqrt{(P \cdot V[[X]])}$ - say $f(X)^{k} \in P \cdot V[[X]]$. Then $f(X)^{k}=$ $r g(X)$ where $g(X) \in V[[X]]$ and $r \in P$, implying that $r \in M_{\lambda_{1}}$ for some $\lambda_{1} \in A$. Thus $f(X)^{k}=r g(X) \in M_{\lambda_{1}}[[X]]$, implying that $f(X) \in M_{\lambda_{1}}[[X]]$. There exists $\lambda_{2} \in \Lambda$ such that $M_{\lambda_{1}} \subset M_{\lambda_{2}}$. Let $s \in M_{\lambda_{2}}, s \notin M_{\lambda_{1}}$; then $(s) \supseteqq M_{\lambda_{1}} \supseteqq A_{f}$, so that $f(X)=\operatorname{sh}(X)$ where $h(X) \in V[[X]]$. Since $s \in M_{\lambda_{2}}, s \in P$; hence $f(X)=\operatorname{sh}(X) \in P \cdot V[[X]]$.

COROLlaRY 4.6. Let $V$ be a valuation ring having a proper prime ideal $P$. If $P$ is idempotent, then there is a prime ideal $Q$ of $V[[X]]$ satisfying $P \cdot V[[X]] \subseteq Q \subset P[[X]]$.

Proof. Theorem 4.5 assures that

$$
\sqrt{(P \cdot V[[X]])}=P \cdot V[[X]] \subset P[[X]] \cdot
$$

Hence there is a prime ideal $Q$ of $V[[X]]$ satisfying $Q \supseteq P \cdot V[[X]]$, $Q \nsupseteq P[[X]]$. Theorem 4.3 then asserts that $Q \subset P[[X]]$.

THEOREM 4.7. Let $V$ be a valuation ring of rank $n$ having $k$ distinct idempotent proper prime ideals. Then $\operatorname{dim} V[[X]] \geqq n+k+1$.

Proof. We use induction on $n$, the case $n=1$ following from Theorem 2.7 and Theorem 3.4.

Assuming the result for valuation rings of rank $t$, let $V$ be a valuation ring of rank $t+1$ having $k$ distinct idempotent proper prime ideals and let $(0) \subset P_{1} \subset P_{2} \subset \cdots \subset P_{t+1}$ be the chain of nonunit prime ideals of $V$. We consider two cases.

Case 1. $P_{1}$ is not idempotent. Here $V / P_{1}$ is a valuation ring of rank $t$ which has $k$ distinct idempotent proper prime ideals. By the induction hypothesis, $\operatorname{dim}\left(V / P_{1}\right)[[X]] \geqq t+k+1$. Since $\left(V / P_{1}\right)[[X]] \cong$ $V[[X]] / P_{1}[[X]]$, this implies that the depth of $P_{1}[[X]]$ is at least $t+k+1$. Since $P_{1}[[X]] \neq(0), \operatorname{dim} V[[X]] \geqq t+k+2$.

Case 2. $P_{1}$ is idempotent. Here $V / P_{1}$ is a valuation ring of rank 
$t$ which has $k-1$ distinct idempotent proper prime ideals. By the induction hypothesis. $\operatorname{dim}\left(V / P_{1}\right)[[X]] \geqq t+(k-1)+1=t+k$; hence the depth of $P_{1}[[X]]$ is at least $t+k$. Since $P_{1}$ is idempotent, Corollary 4.6 asserts that there is a prime ideal $Q$ of $V[[X]]$ satisfying $P_{1} \cdot V[[X]] \subseteq Q \subset P_{1}[[X]]-$ in particular, $(0) \subset Q \subset P_{1}[[X]]$. Since the depth of $P_{1}[[X]]$ is at least $t+k$, we see that $\operatorname{dim} V[[X]] \geqq$ $t+k+2$.

Lemma 4.8. Let $V$ be valuation ring and let $P$ be a proper prime ideal of $V$.

(a) If $Q^{\prime}$ is a prime ideal of $V_{P}[[X]]$ which satisfies $\left(P V_{P}\right) \cdot$ as $V_{P}[[X]] \subseteq Q^{\prime} \subset\left(P V_{P}\right)[[X]]$, then $Q^{\prime}$ is a prime ideal of $V[[X]]$ which satisfies $P \cdot V[[X]] \leqq Q^{\prime} \subset P[[X]]$.

(b) Conversely, if $Q$ is a prime ideal of $V[[X]]$ which satisfies $P \cdot V[[X]] \cong Q \subset P[[X]]$, then $Q$ is a prime ideal of $V_{P}[[X]]$ which satisfies $\left(P V_{P}\right) \cdot V_{P}[[X]] \subseteq Q \subset\left(P V_{P}\right)[[X]]$.

Proof. To establish (a), we observe that $Q^{\prime} \subseteq\left(P V_{P}\right)[[X]]=$ $P[[X]] \subseteq V[[X]]$, whereby $Q^{\prime} \cap V[[X]]=Q^{\prime}$.

We now establish $(b)$; we begin by proving that $Q$ is an ideal of $V_{P}[[X]]$. Let $f(X) \in Q$ and $g(X) \in V_{P}[[X]]$; we show that $f(X) \cdot$ as $g(X) \in Q$. Choose $h(X) \in P[[X]], h(X) \notin Q$. For each $i, j \in \omega_{0}, g_{i} \in V_{P}$ and $h_{j} \in P$, implying that $g_{i} h_{j} \in P V_{P}=P$. Hence $g(X) h(X) \in P[[X]] \subseteq$ $V[[X]]$, implying that $f(X)[g(X) h(X)] \in Q$. Since $f(X) \in Q \subseteq P[[X]]$, each $f_{i} \in P$; hence $f(X) g(X) \in P[[X]] \subseteq V[[X]]$. Since $[f(X) g(X)]$. $h(X) \in Q$ where $f(X) g(X) \in V[[X]], h(X) \in V[[X]]$, and $h(X) \notin Q$, we conclude that $f(X) g(X) \in Q$. Hence $Q$ is an ideal of $V_{P}[[X]]$.

We now prove that $Q$ is a prime ideal of $V_{P}[[X]]$. Let $S=$ $V[[X]] \backslash Q$; then $S$ is a multiplicative system in $V[[X]]$, hence also in $V_{P}[[X]]$, and $S$ clearly does not meet the ideal $Q$ of $V_{P}[[X]]$. Hence there is a prime ideal $Q^{*}$ of $V_{P}[[X]]$ which satisfies $Q \subseteq Q^{*}, Q^{*} \cap S=\varnothing$. Since $Q \subseteq Q^{*}, Q \subseteq Q^{*} \cap V[[X]]$; since $Q^{*} \cap S=\varnothing, Q^{*} \cap V[[X]] \subseteq Q$. Thus $Q^{*} \cap V[[X]]=Q$. Observe now that $Q^{*} \supseteqq Q \supseteqq P \cdot V[[X]]=$ $\left(P V_{P}\right) \cdot V_{P}[[X]]$. By Theorem 4.3, $Q^{*}$ compares with $\left(P V_{P}\right)[[X]]=$ $P[[X]]$. Since $Q^{*}$ lies over $Q$ we must have that $Q^{*} \subset P[[X]] \subseteq V[[X]]$, implying that $Q^{*}=Q$. Hence $Q$ is a prime ideal of $V_{P}[[X]]$.

That $\left(P V_{P}\right) \cdot V_{P}[[X]] \subseteq Q \subset\left(P V_{P}\right)[[X]]$ is clear.

THEOREM 4.9. The following conditions are equivalent:

(a) If $V$ is a rank one nondiscrete valuation ring, then $V[[X]]$ has finite dimension.

(b) If $V$ is a valuation ring having finite rank $n$, then $V[[X]]$ has finite dimension. 
Proof. It is clear that (b) $\rightarrow(a)$. We prove that $(a) \rightarrow(b)$ using induction on $n$, the case $n=1$ being a consequence of (a) and Theorem 2.7.

We now assume that if $W$ is a valuation ring of rank $k$, then $W[[X]]$ has finite dimension. Let $V$ be a valuation ring of rank $k+1$ which has minimal prime $P_{1}$. Let $(0) \subset Q_{1} \subset Q_{2} \subset \cdots \subset Q_{t}$ be a chain of prime ideals of $V[[X]]$. Let $d=\operatorname{dim} V_{P_{1}}[[X]]$. Corollary 2.3 assures that there are at most $d$ proper prime ideals in this chain which contract to $(0)$ in $V$. Choose $m$ so that $Q_{m} \cap V=(0)$ and $Q_{m+1} \cap V \neq(0)$; then $m \leqq d$. For $r \geqq m+1, Q_{r} \cap V \supseteqq P_{1}$; Theorem 4.3 assures that for $r \geqq m+1, Q_{r}$ compares with $P_{1}[[X]]$. Lemma 4.8 assures that at most $d$ of the ideals $Q_{m+1}, Q_{m+2}, \cdots, Q_{t}$ are contained in $P_{1}[[X]]$, whereby $Q_{m+d+1} \supset P_{1}[[X]]$. Since $m \leqq d$, we have that $Q_{2 d+1} \supseteqq Q_{m+d+1} \supset P_{1}[[X]]$.

By the induction hypothesis, $\left(V / P_{1}\right)[[X]]$ has finite dimension. The depth of $P_{1}[[X]]$ is at most $\left(\operatorname{dim}\left(V / P_{1}\right)[[X]]-1\right)$. It follows that the depth of $Q_{2 d+1}$ is at most $\left(\operatorname{dim}\left(V / P_{1}\right)[[X]]-1\right)$, whereby

$$
t \leqq(2 d+1)+\left(\operatorname{dim}\left(V / P_{1}\right)[[X]]-1\right)=2 d+\operatorname{dim}\left(V / P_{1}\right)[[X]] \cdot
$$

We conclude that $\operatorname{dim} V[[X]] \leqq 2 d+\operatorname{dim}\left(V / P_{1}\right)[[X]]$, whereby $V[[X]]$ has finite dimension.

THEOREM 4.10. The following conditions are equivalent:

(a) If $V$ is a rank one nondiscrete valuation ring, then the ascending chain condition for prime ideals holds in $V[[X]]$.

(b) If $V$ is a valuation ring having finite rank $n$, then the ascending chain condition for prime ideals holds in $V[[X]]$.

The proof of Theorem 4.10 is analogous to the proof of Theorem 4.9 and will therefore be omitted.

Added in proof. Jimmy T. Arnold has recently conveyed to me a paper of his, On Krull Dimension in Power Series Rings, in which he has established the following result.

Let $R$ be a commutative ring with identity. If there exists a prime ideal $P$ of $R$ such that $\sqrt{(P \cdot R[[X]])} \neq P[[X]]$, then $R[[X]]$ has infinite dimension.

It follows immediately that if $V$ is a valuation ring which is not discrete, then $V[[X]]$ has infinite dimension. 


\section{REFERENCES}

1. Robert Gilmer, Multiplicative ideal theory, Queen's Papers on Pure and Applied Mathematics, No. 12, Kingston, Ontario, 1968.

2. Kenkichi Iwasawa, On linearly ordered groups, J. Math. Soc. Japan 1 (1948), 1-9. 3. A. Seidenberg, $A$ note on the dimension theory of rings, Pacific J. Math. 3 (1953), 505-512.

4. - On the dimension theory of rings, Pacific J. Math. 4 (1954), 603-614.

5. O. Zariski and P. Samuel, Commutative Algebra, Vol. 1, Princeton, 1958.

Received May 12, 1970. This paper is part of the author's dissertation, written under the direction of Professor Robert Gilmer of Florida State University.

STETSON UNIVERSITY AND

FLORIDA STATE UNIVERSITY 



\section{PACIFIC JOURNAL OF MATHEMATICS}

\section{EDITORS}

\author{
H. SAMELSON \\ Stanford University \\ Stanford, California 94305 \\ Richard Pierce \\ University of Washington \\ Seattle, Washington 98105
}

\author{
J. DugundJI \\ Department of Mathematics \\ University of Southern California \\ Los Angeles, California 90007 \\ RICHARD ARENS \\ University of California \\ Los Angeles, California 9.0024
}

\section{ASSOCIATE EDITORS}

\begin{tabular}{|c|c|}
\hline E. F. BECKENBACH & K. YoshidA \\
\hline \multicolumn{2}{|c|}{ SUPPORTING INSTITUTIONS } \\
\hline UNIVERSITY OF BRITISH COLUMBIA & STANFORD UNIVERSITY \\
\hline CALIFORNIA INSTITUTE OF TECHNOLOGY & UNIVERSITY OF TOKYO \\
\hline UNIVERSITY OF CALIFORNIA & UNIVERSITY OF UTAH \\
\hline MONTANA STATE UNIVERSITY & WASHINGTON STATE UNIVERSITY \\
\hline UNIVERSITY OF NEVADA & UNIVERSITY OF WASHINGTON \\
\hline NEW MEXICO STATE UNIVERSITY & * \\
\hline OREGON STATE UNIVERSITY & AMERICAN MATHEMATICAL SOCIETY \\
\hline UNIVERSITY OF OREGON & CHEVRON RESEARCH CORPORATION \\
\hline OSAKA UNIVERSITY & TRW SYSTEMS \\
\hline UNIVERSITY OF SOUTHERN CALIFORNIA & NAVAL WEAPONS CENTER \\
\hline
\end{tabular}

The Supporting Institutions listed above contribute to the cost of publication of this Journal, but they are not owners or publishers and have no responsibility for its content or policies.

Mathematical papers intended for publication in the Pacific Journal of Mathematics should be in typed form or offset-reproduced, (not dittoed), double spaced with large margins. Underline Greek letters in red, German in green, and script in blue. The first paragraph or two must be capable of being used separately as a synopsis of the entire paper. The editorial "we" must not be used in the synopsis, and items of the bibliography should not be cited there unless absolutely necessary, in which case they must be identified by author and Journal, rather than by item number. Manuscripts, in duplicate if possible, may be sent to any one of the four editors. Please classify according to the scheme of Math. Rev. Index to Vol. 39. All other communications to the editors should be addressed to the managing editor, Richard Arens, University of California, Los Angeles, California, 90024.

50 reprints are provided free for each article; additional copies may be obtained at cost in multiples of 50 .

The Pacific Journal of Mathematics is published monthly. Effective with Volume 16 the price per volume (3 numbers) is $\$ 8.00$; single issues, $\$ 3.00$. Special price for current issues to individual faculty members of supporting institutions and to individual members of the American Mathematical Society: $\$ 4.00$ per volume; single issues $\$ 1.50$. Back numbers are available.

Subscriptions, orders for back numbers, and changes of address should be sent to Pacific Journal of Mathematics, 103 Highland Boulevard, Berkeley, California, 94708.

PUBLISHED BY PACIFIC JOURNAL OF MATHEMATICS, A NON-PROFIT CORPORATION

Printed at Kokusai Bunken Insatsusha (Internatıonal Academic Printing Co., Ltd.), 7-17, Fujimi 2-chome, Chiyoda-ku, Tokyo, Japan. 


\section{Pacific Journal of Mathematics}

\section{Vol. 35, No. $3 \quad$ November, 1970}

John D. Arrison and Michael Rich, On nearly commutative degree one algebras . . . 533

Bruce Alan Barnes, Algebras with minimal left ideals which are Hilbert spaces . . . . 537

Robert F. Brown, An elementary proof of the uniqueness of the fixed point index . . . 549

Ronn L. Carpenter, Principal ideals in F-algebras .................... 559

Chen Chung Chang and Yiannis (John) Nicolas Moschovakis, The Suslin-Kleene

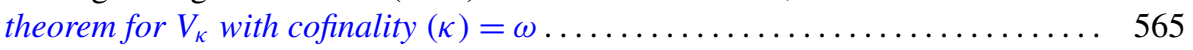

Theodore Seio Chihara, The derived set of the spectrum of a distribution

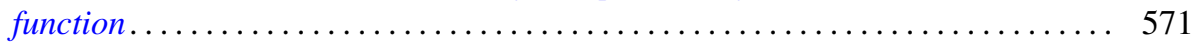

Tae Geun Cho, On the Choquet boundary for a nonclosed subspace of $C(S) \ldots \ldots \quad 575$

Richard Brian Darst, The Lebesgue decomposition, Radon-Nikodym derivative,

conditional expectation, and martingale convergence for lattices of sets .......

David E. Fields, Dimension theory in power series rings . . . . . . . . . . . .

Michael Lawrence Fredman, Congruence formulas obtained by counting

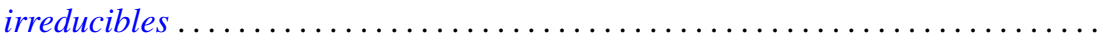

John Eric Gilbert, On the ideal structure of some algebras of analytic functions.....

G. Goss and Giovanni Viglino, Some topological properties weaker than

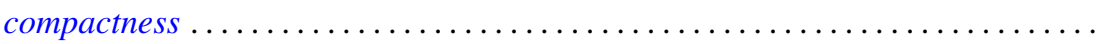

581

601

625

George Grätzer and J. Sichler, On the endomorphism semigroup (and category) of

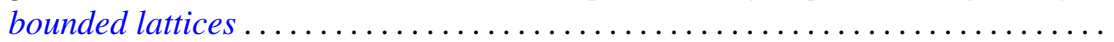

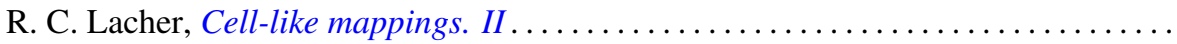

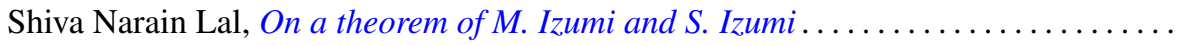

661

Howard Barrow Lambert, Differential mappings on a vector space ...............

Richard G. Levin and Takayuki Tamura, Notes on commutative power joined

semigroups.

Robert Edward Lewand and Kevin Mor McCrimmon, Macdonald's theorem for quadratic Jordan algebras.

J. A. Marti, On some types of completeness in topological vector spaces ....

Walter J. Meyer, Characterization of the Steiner point

717

Saad H. Mohamed, Rings whose homomorphic images are $q$-rings ...

727

Thomas V. O'Brien and William Lawrence Reddy, Each compact orientable surface

of positive genus admits an expansive homeomorphism ...

737

Robert James Plemmons and M. T. West, On the semigroup of binary relations...

743

Calvin R. Putnam, Unbounded inverses of hyponormal operator . .

755

William T. Reid, Some remarks on special disconjugacy criteria for differential

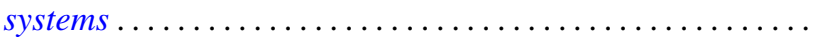

C. Ambrose Rogers, The convex generation of convex Borel sets in euclidean

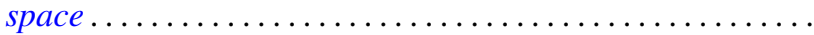

S. Saran, A general theorem for bilinear generating functions .

S. W. Smith, Cone relationships of biorthogonal systems ......

Wolmer Vasconcelos, On commutative endomorphism rings ....

795

Vernon Emil Zander, Products of finitely additive set functions from Orlicz

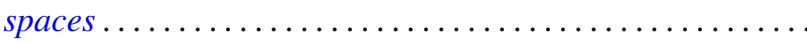

G. Sankaranarayanan and C. Suyambulingom, Correction to: "Some renewal

theorems concerning a sequence of correlated random variables" .

Joseph Zaks, Correction to: "Trivially extending decompositions of $E^{n}$ ”....... 805

Dong Hoon Lee, Correction to: "The adjoint group of Lie groups" ............ 805

James Edward Ward, Correction to: "Two-groups and Jordan algebras". 